\title{
Nanoparticles Based on Linear and Star-Shaped Poly(Ethylene Glycol)-Poly( $\varepsilon$-Caprolactone) Copolymers for the Delivery of Antitubulin Drug
}

\author{
Karim S. Shalaby' - Mahmoud E. Soliman' $\cdot$ Giulia Bonacucina ${ }^{2} \cdot$ Marco Cespi $^{2} \cdot$ \\ Giovanni F. Palmieri $^{2}$ - Omaima A. Sammour' ${ }^{\prime}$ Abdelhameed A. El Shamy' ${ }^{\prime}$ Lisbeth Illum ${ }^{3}$. \\ Luca Casettari $^{4}$
}

Received: 30 December 2015 / Accepted: 29 April 2016

(C) Springer Science+Business Media New York 2016

\begin{abstract}
Purpose Biodegradable polymeric nanoparticles of different architectures based on polyethylene glycol-co-poly $(\varepsilon-$ caprolactone) block copolymers have been loaded with noscapine (NOS) to study their effect on its anticancer activity. It was intended to use solubility of NOS in an acidic environment and ability of the nanoparticles to passively target drugs into cancer tissue to modify the NOS pharmacokinetic properties and reduce the requirement for frequent injections.

Methods Linear and star-shaped copolymers were synthetized and used to formulate NOS loaded nanoparticles. Cytotoxicity was performed using a sulforhodamine B method on MCF-7 cells, while biocompatibility was determined on rats followed by hematological and histopathological investigations.
\end{abstract}

Results Formulae with the smallest particle sizes and adequate entrapment efficiency revealed that NOS loaded nanoparticles showed higher extent of release at $\mathrm{pH}$ 4.5. Colloidal stability suggested that nanoparticles would be stable in blood when injected into the systemic circulation. Loaded nanoparticles had $\mathrm{IC}_{50}$ values lower than free drug. Hematological

Electronic supplementary material The online version of this article (doi: | 0. I007/s I |095-0 | 6- 1939-8) contains supplementary material, which is available to authorized users.

Luca Casettari

luca.casettari@uniurb.it

Department of Pharmaceutics and Industrial Pharmacy, Faculty of Pharmacy, Ain Shams University, I 1566 Cairo, Egypt

2 School of Pharmacy, University of Camerino, Via S. Agostino I, 62032 Camerino, MC, Italy

3 IDentity, 19 Cavendish Crescent North, The Park, Nottingham NG7 IBA, UK

4 Department of Biomolecular Sciences, School of Pharmacy, University of Urbino, Piazza Rinascimento, 6, 61029 Urbino, PU, Italy and histopathological studies showed no difference between treated and control groups. Pharmacokinetic analysis revealed that formulation P1 had a prolonged half-life and better bioavailability compared to drug solution.

Conclusions Formulation of NOS into biodegradable polymeric nanoparticles has increased its efficacy and residence on cancer cells while passively avoiding normal body tissues.

KEY WORDS noscapine $\cdot$ passive targeting $\cdot \mathrm{pH}$ dependent release $\cdot$ polyethylene glycol-co-poly( $\varepsilon$-caprolactone)

(PEG-CO-PCL) · polymeric nanoparticles

$\begin{array}{ll}\text { ABBREVIATIONS } \\ \text { AFM } & \text { Atomic force microscopy } \\ \text { CMC } & \text { Critical micelle concentration } \\ \text { DLS } & \text { Dynamic light scattering } \\ \text { HPLC } & \text { High performance liquid chromatography } \\ \text { NOS } & \text { Noscapine } \\ \text { NPS } & \text { Nanoparticles } \\ \text { PEG-co-PCL } & \begin{array}{l}\text { Polyethylene glycol-co-poly(e-caprolactone) } \\ \text { block copolymers }\end{array} \\ \text { PLA } & \text { Polyllactic acid) } \\ \text { PLGA } & \text { Polyllactide-co-glycolide) } \\ \text { RES } & \text { Reticuloendothelial system } \\ \text { SRB } & \text { Sulphorhodamine-B }\end{array}$

\section{INTRODUCTION}

Therapeutic strategies for most cancers, including breast cancer, currently involve a combination of surgery, radiotherapy, immunotherapy, hormone therapy and/or chemotherapy. The efficacy of a therapeutic formulation depends on its ability to selectively target diseased tissue, overcome biological 
barriers, and intelligently respond to the disease environment to release therapeutic agents (1).

Chemotherapy for the treatment of cancer refers to the use of chemotherapeutical agents to disrupt the normal functioning of a cell by inducing apoptosis or preventing replication. The limitations of conventional chemotherapeutic agents are mainly due to their nonspecific distribution in the body where they affect both cancerous and normal cells, thereby reducing the achievable dose within the tumor tissues and causing excessive toxicities to normal cells. Moreover, cancer cells can develop a resistance to the cytotoxicity not only of conventional chemotherapeutics but also of newer molecularly targeted therapeutics (2).

The development of controlled release technology and targeted drug delivery may result in a more efficient and less harmful solution to overcome the limitations found in conventional chemotherapy. Recent studies have been focused on developing nanoscale delivery vehicles capable of controlling the release of chemotherapeutic agents directly inside cancer cells $(3,4)$. Micellar structures are well suited to drug delivery applications for multiple reasons. Their diameters are generally less than $100 \mathrm{~nm}$, permitting them to extravasate through the fenestrations in tumor vessels $(1,5-7)$. The fenestration size varies depending on the type of tumor e.g. for malignant brain tumors it ranges from 7 to $100 \mathrm{~nm}$, however for peripheral tumors it ranges from $200 \mathrm{~nm}$ to $1.2 \mu \mathrm{m}$ (5). Large pore cut off sizes $(\sim 700 \mathrm{~nm})$ were reported for breast cancer models $(8)$. Moreover, the hydrophilic surfaces of micellar structures based on amphiphiles comprising PEG may shield them from immediate recognition by the reticuloendothelial system (RES) leading to an increase in their circulation time (9). The core of the micellar structure can load hydrophobic drugs which are protected by the hydrophilic corona during transport to the tumor site (10). Many micellar formulations are currently under clinical evaluation for the treatment of cancer (e.g. doxorubicin loaded micelles, paclitaxel loaded polymeric micelles and epirubicin loaded micelles (11-14)).

Previous studies on micellar like nanoparticles (NPs) for drug targeting, have focused mainly on the self-assembly of linear di- or tri-block amphiphilic copolymers into structures with a hydrophobic core and a hydrophilic exterior $(15,16)$. Such NPs have been based on a range of polymers of different hydrophobicity such as poly(lactic acid) (PLA), poly (amineesters) (PAE), poly( $\varepsilon$-caprolactone) (PCL), and poly(lactide-coglycolide) PLGA linked to a hydrophilic counterpart, especially PEG (15,17-21). However, although stable in solution, such NP structures based on linear copolymers in general suffer from low encapsulation efficiency and difficulty in controlling polymer degradation (22).

During the last decade, the synthesis of more complicated grafted, star-shaped, Y-shaped and dumbbell-shaped or dendritic copolymer structures containing three or more hydrophilic arms emerging from the polymer center have been carried out and the copolymers used for production of selfassembling NPs for targeted drug delivery (4,23-25).

The copolymers used in these studies have most often been based on PLA or PLGA but star-shaped block copolymers based on PCL have also been produced (26,27).

NPs produced from star-shaped structures have been reported to have superior properties, e.g. in overcoming the thermodynamic instability of in the blood stream, showing better loading capabilities, as compared to NPs produced from linear di- or tri-block copolymers (26,28-30). Moreover star-shaped polymers showed higher host-guest interactions with small drug molecules when compared to linear analogues (31). However, systematic comparisons between NPs made from copolymers with different topologies and physicochemical characteristics have only rarely been reported (25) and very little has been reported in this regard on PCL.

It has been shown that the in vivo circulation time of NPs made from block-copolymers by the nanoprecipitation method (also used in the present work) is dependent on a range of factors related to the physicochemical characteristics of the copolymer (32). Hence, it was found that the circulation time was inversely dependent on the molecular weight of the hydrophobic block, directly dependent on the log P of the hydrophobic block and the molecular weight of the PEG chain. Further, it was shown that crystallization of the hydrophobic block disrupts the PEG surface coverage and hence may influence the NP circulation time.

PCL is a biodegradable polyester with a hydrophobic part in its backbone that degrade very slowly under hydrolytic conditions in the human body. It has been shown to have a $\log \mathrm{P}$ between 11 and 12 for molecular weights between 3 and $9 \mathrm{kDa}$, respectively (32). In comparison, PLGA (50/50) showed a $\log \mathrm{P}$ between 2 and 4 for molecular weights between 5 and $8 \mathrm{kDa}$ and PLA showed $\mathrm{log} \mathrm{P}$ between 7 and 11 for molecular weights between 3 and $10 \mathrm{kDa}$. Hence, PCL has a superior hydrophobicity to PLGA and PLA. It has further been shown that the crystallinity of PCL increases with increase in molecular weight (32). Block copolymer of PEG with PCL are expected to enhance the hydrophilicity of NP, prevent opsonization, and phagocytosis by the RES system due to steric stabilization created by PEG on the surface $(33,34)$. It was therefore of interest to investigate the physicochemical properties, cytotoxicity, acute toxicity and pharmacokinetics of NPs made from PCL of different molecular weight and different copolymer structures, including di-, tri-block, star shaped and dumbbell shaped copolymers, to include their interaction with and release of a model cancer drug noscapine.

Noscapine (NOS) is a phthalide isoquinoline alkaloid derived from opium. It has been used as an oral antitussive agent and has shown minimal toxic effects in animals and humans. However, it was found to have a tubulin-binding activity and 
currently is undergoing clinical trials for anticancer therapy (35). Although NOS has many advantages, such as higher solubility in acidic medium (favored release near cancer tissues) and resistance free anticancer properties, it has also several limitations like other cancer chemotherapeutic drugs. These limitations include: (a) hydrophobicity and limited solubility in an aqueous phase (36); (b) lack of selectivity, as most anticancer drugs, which may cause significant damage to rapidly proliferating normal cells (35); (c) low oral bioavailability (about 31\%) both in humans and mice (37); (d) short plasma half-life of below $2 \mathrm{~h}$, which makes the design of controlled release formulations difficult (36); (f) rapid elimination from body tissues, hence, multiple injections are required for successive chemotherapy (38). In addition, the selective release of NOS as an alkaloid in the acidic medium of cancer tissue would increase its safety and efficacy during use in anticancer therapies.

The aim of the work carried out in this paper was to evaluate, in a systematic study, the effect of different factors such as molecular weight of PCL, the PCL-PEG copolymer structure (di-, tri-block, star-shaped or dumbbell-shaped), the CMC and the polymer to drug ratio on the resultant NP size, zeta potential, drug loading and entrapment efficiencies. From this work the most promising structures will be selected and further characterized in terms of atomic force microscopy, colloidal stability, cytotoxic activity, acute toxicity, hematological and histopathological toxicity in rats and pharmacokinetics of the NOS in order to fully understand the importance of the different variables on the suitability of the PCL-PEG structures for drug targeting of cancer drug. In addition, the selective release of NOS will be evaluated in vitro at different $\mathrm{pH}$ values to mimic the release in the blood stream and in cancerous tissues.

\section{MATERIALS AND METHODS}

\section{Materials}

mPEG 1.9 and PEG 2 kDa were purchased from Polysciences (Eppelheim, Germany). Stannous-2-ethyl-hexanoate and $\varepsilon$ caprolactone were purchased from Sigma-Aldrich (Milan, Italy). Noscapine (NOS) was a gift sample from EIPICO Pharmaceutical Company (El Asher of Ramadan, Egypt). Acetone, methanol, disodium hydrogen phosphate dihydrate, and potassium dihydrogen phosphate were purchased from El Nasr Pharmaceutical Company (Cairo, Egypt). Chloroform, dichloromethane (DCM), acetonitrile, tetrahydrofuran (THF), deuterated chloroform $\left(\mathrm{CDCl}_{3}\right)$, and diethyl ether were purchased from Thermo Fisher scientific Inc. (Massachusetts, USA).

Fetal Bovine Serum (FBS), penicillin-streptomycin, trypsinEDTA, Tris-HCl, and RPMI 1640 medium were purchased from Lonza (New Jersy, USA). Sulphorhodamine-B, trichloroacetic acid, Leishman's stain, dialysis membrane, hematoxylin and eosin were purchased from Sigma-Aldrich (St. Louis, USA). HPLG internal standard papaverine was obtained from Recordati (Milan, Italy). Muscovite mica discs were purchased from Agar Scientific (Stansted, UK).

\section{Methods}

\section{Synthesis and Characterizations of Block Copolymers}

Synthesis of block copolymers (i.e. mPEG-PCL, PCL-PEGPCL, 4-armed PEG-PCL and 6-armed PEG-PCL) (Fig. 1) was done by the ring opening polymerization method as previously described $(4,26,30,31)$. The characteristics of the synthesized polymers are given in Table 1 . Characterization of synthesized block copolymers was done using $\mathrm{H}^{1}$-NMR and GPC analysis (supplementary information). Volume fraction of PEG ( $f_{\text {PEG}}$ ) was calculated using Mn (NMR) and the densities of bulk polymers (density of $\mathrm{PEG}=1.13 \mathrm{~g} \mathrm{~cm}^{-3}$, density of PCL $=1.25 \mathrm{~g} \mathrm{~cm}^{-3}$ ) using the following equation (Eq. 1) (36):

$f P E G=\frac{\frac{\text { the mass of } P E G}{\text { the density of } P E G}}{\frac{\text { the mass of } P E G}{\text { the density of } P E G}+\frac{\text { the mass of } P C L}{\text { the density of } P C L}}$

\section{Preparation of NOS Loaded Polymeric NPs}

NPs were prepared using the nanoprecipitation method previously described by Fessi et al. (37). The polymer and NOS in different ratios (10:1, 5:1 and 2.5:1) were accurately weighed and dissolved in acetone $(1 \mathrm{~mL})$. The organic phase was added dropwise to deionized water $(10 \mathrm{ml})$ and stirred magnetically at $1000 \mathrm{rpm}$ until complete evaporation of acetone. A list of the nanoparticles prepared from the polymers described in Table 1 is given in Table 2. The produced nanoparticles were centrifuged at 15,000 rpm for $30 \mathrm{~min}$ then washed twice using deionized water and frozen to $-52^{\circ} \mathrm{C}$ before application of vacuum $(0.05 \mathrm{mBar})$ to freeze dry them using (Laboratory Freeze Drier Alpha 1-4, Martin Christ, Germany).

\section{Characterization of the Prepared NPs}

Particle Size and Zeta Potential Determinations. Dynamic light scattering (DLS) was used to measure average particle diameter (Z-average) and polydispersity index (PDI) of the prepared NPs in deionized water, while electrophoretic mobility was used to measure zeta potential employing a Zetasizer Nano ZS (Malvern Instruments Ltd., UK) equipped with 4 mW 633 nm He-Ne lasers. Samples were diluted 

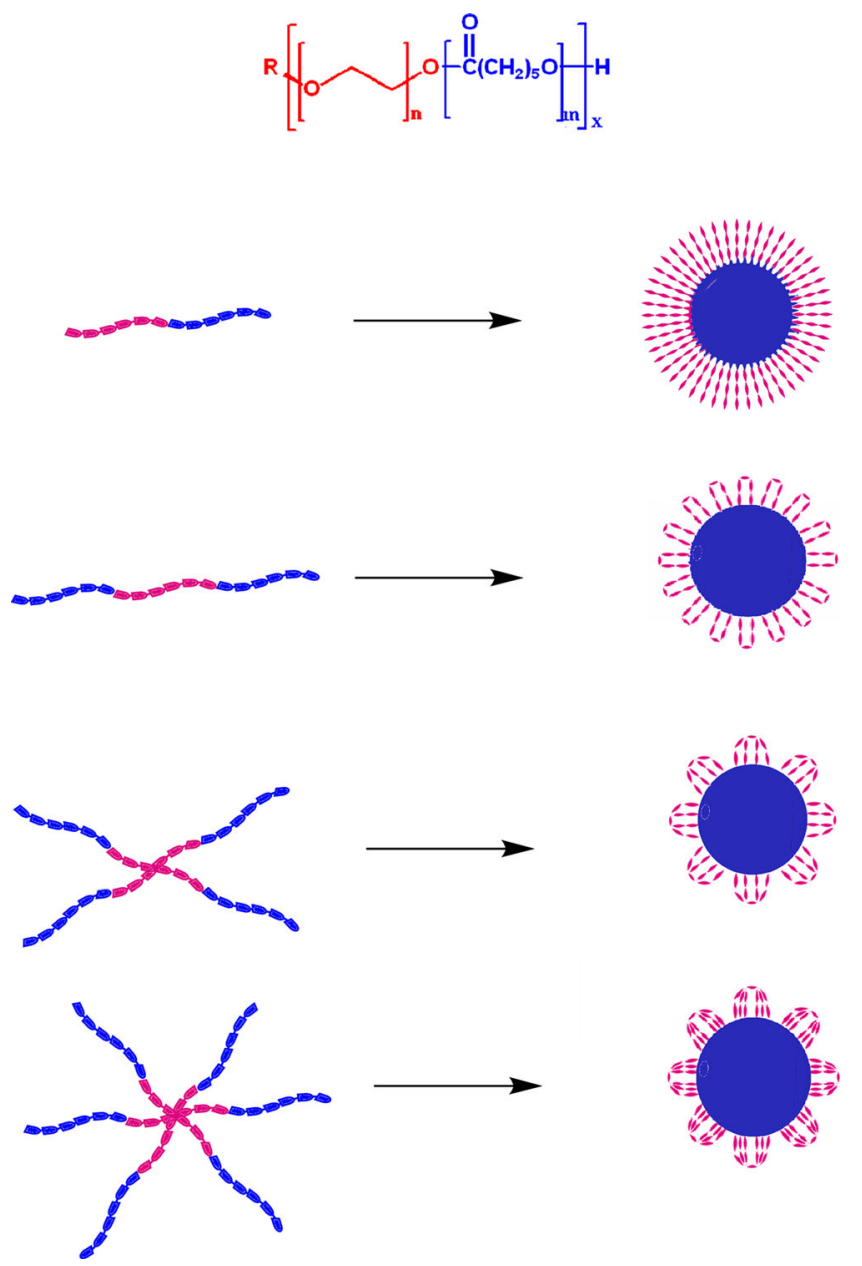

Fig. I Different architecture of poly(ethylene glycol) (Red colored) and poly(caprolactone) (Blue colored) block copolymers.

appropriately using deionized water then samples were equilibrated at $25^{\circ} \mathrm{C}$ in the device sample holder and analyses were performed with the laser fixed at an angle of $173^{\circ}$. Data were obtained and expressed as the average of at least 12 runs and shown as the mean value \pm standard deviation of at least three measurements.

Determination of Loading and Encapsulation Efficiency. NPs were purified by centrifugation twice at 15,000 rpm for $30 \mathrm{~min}$ (Cooling centrifuge, Hermle, Germany), the supernatant was discarded then the sediment was washed using deionized water and freeze dried (Laboratory Freeze Drier Alpha 1-4, Martin Christ, Germany). NOS entrapment efficiency in the NPs was assessed by dissolving a specific weight of NPs in a mixture of chloroform and methanol (1:9) then the NOS concentration was determined at $291 \mathrm{~nm}$ in the organic solution using UV spectroscopy (Shimadzu 1650PC, Tokyo, Japan). The loading efficiency (DL\%) and entrapment efficiency $(\mathrm{EE} \%$ ) was obtained using the following equation (Eq. 2) $(38,39)$ :

$$
\begin{aligned}
& D L(\%)=\frac{\text { Drug loaded }}{\text { Drug loaded }+ \text { polymer }} \times 100 \\
& E E(\%)=\frac{\text { Actual drug loading }}{\text { Theoretical drug loading }} \times 100
\end{aligned}
$$

Critical Micelle Concentration (CMC) Assay. CMC values of P16 micelles were determined with a spectroflurometric technique using pyrene as the fluorescence probe (40). For CMC measurement, micellar dispersions loaded with pyrene were prepared by adding $12 \mu \mathrm{L}$ solution of pyrene in ethanol $(0.1 \mathrm{mg} / \mathrm{mL})$ to $20 \mathrm{~mL}$ vials then leaving ethanol to evaporate for 1 h. $10 \mathrm{~mL}$ of different concentrations $(0.01-20 \mathrm{mg} / \mathrm{L})$ of the prepared polymer solution was added into the specified vial, keeping the pyrene concentration in the vial solution to be $1.2 \times 10^{-4} \mathrm{mg} / \mathrm{mL}$. The stoppered vials were heated for $3 \mathrm{~h}$ at $65^{\circ} \mathrm{C}$ to equilibrate pyrene and the formed micelles were

\begin{tabular}{|c|c|c|c|c|c|c|c|}
\hline \multirow[t]{2}{*}{ Code } & \multirow[t]{2}{*}{ Polymer } & \multicolumn{3}{|c|}{ Molecular weight ( $\mathrm{g} \mathrm{mol}^{-1}$ ) } & \multirow[t]{2}{*}{$\mathrm{Mw} / \mathrm{Mn}(\mathrm{GPC})$} & \multirow{2}{*}{$\begin{array}{l}f_{\text {PEG }} \\
(\mathrm{NMR})\end{array}$} & \multirow{2}{*}{$\begin{array}{l}\text { CMC } \\
(\mathrm{mg} / \mathrm{L})\end{array}$} \\
\hline & & Mwt $_{(N M R)}$ & PCL block (NMR) & $M n_{(G P C)}$ & & & \\
\hline $\mathrm{Pl}$ & mPEGI.9 kDa-PCL & 3154 & 1254 & 3520 & 1.7 & 0.63 & 7.08 \\
\hline P2 & mPEGI.9 kDa-PCL & 5662 & 3762 & 5953 & 1.9 & 0.36 & 5.62 \\
\hline P3 & PCL-PEG2.0 kDa-PCL & 5150 & 1575 & 5391 & 1.6 & 0.41 & 4.90 \\
\hline P4 & PCL-PEG2.0 kDa-PCL & 8263 & 3132 & 8677 & 2.4 & 0.26 & 1.26 \\
\hline P5 & 4 Arms PEG I OkDa -(PCL) & 20,348 & 2587 & 21,852 & 1.8 & 0.52 & 0.49 \\
\hline P6 & 6Arms PEG I 7kDa -(PCL)6 & 33,230 & 2705 & 41,717 & 1.5 & 0.54 & 1.58 \\
\hline
\end{tabular}

Table I The Molecular Weights and Volume Fraction of PEG of Block Co-polymers Used in the Preparation of Nanoparticles

Mwt the Molecular weight of polymer

$M_{w}$ Weight-average molecular weight

$M_{n}$ Number-average molecular weight

$\mathrm{Mw} / \mathrm{Mn}$ Poly dispersity index

$f_{P E G}$ Volume fraction of PEG 
Table 2 Particle Size, Poly Dispersity Index (PDI) and Zeta Potential of Prepared Nanoparticles

\begin{tabular}{|c|c|c|c|c|c|c|}
\hline NPs & Polymer:drug ratio & Size \pm SD & $\mathrm{PDI} \pm \mathrm{SD}$ & Zeta \pm SD & $\mathrm{EE} \% \pm \mathrm{SD}$ & $\mathrm{DL} \% \pm \mathrm{SD}$ \\
\hline \multirow[t]{3}{*}{ NOS-PI } & $10: 1$ & $89.79 \pm 4.12^{*}$ & $0.442 \pm 0.027$ & $-15.50 \pm 0.46$ & $20.20 \pm 2.78^{*}$ & $1.84 \pm 0.25$ \\
\hline & $5: 1$ & $93.16 \pm 9.18^{*}$ & $0.415 \pm 0.061$ & $-19.30 \pm 2.25$ & $12.86 \pm 1.96$ & $2.14 \pm 0.33$ \\
\hline & $2.5: 1$ & $131.23 \pm 21.63$ & $0.38 \pm 0.043$ & $-19.87 \pm 0.50$ & $13.62 \pm 1.99$ & $3.89 \pm 0.57$ \\
\hline \multirow[t]{3}{*}{ NOS-P2 } & $10: 1$ & $173.15 \pm 3.18^{*}$ & $0.61 \pm 0.103$ & $-11.88 \pm 9.79$ & $37.61 \pm 1.61^{*}$ & $3.42 \pm 0.15$ \\
\hline & $5: 1$ & $201.27 \pm 5.80 *$ & $0.732 \pm 0.007$ & $-11.17 \pm 0.67$ & $25.62 \pm 6.11^{*}$ & $4.27 \pm 1.02$ \\
\hline & $2.5: 1$ & $245.97 \pm 18.85$ & $0.470 \pm 0.069$ & $-12.43 \pm 6.52$ & $15.18 \pm 1.97$ & $4.34 \pm 0.56$ \\
\hline \multirow[t]{3}{*}{ NOS-P3 } & $10: 1$ & $275.70 \pm 6.08$ & $0.50 \pm 0.167$ & $-10.94 \pm 1.36$ & $29.74 \pm 15.95$ & $2.7 \pm 1.54$ \\
\hline & $5: 1$ & $387.97 \pm 5.7 I^{*}$ & $0.513 \pm 0.135$ & $-|1.63 \pm 1.5|$ & $42.10 \pm 2.19$ & $7.02 \pm 0.37$ \\
\hline & $2.5: 1$ & $261.43 \pm 48.75$ & $0.690 \pm 0.141$ & $-11.15 \pm 2.09$ & $24.87 \pm 3.17$ & $7.11 \pm 0.91$ \\
\hline \multirow[t]{3}{*}{ NOS-P4 } & $10: 1$ & $65.61 \pm 6.53^{*}$ & $0.45 \pm 0.054$ & $-17.55 \pm 1.48$ & $34.64 \pm 4.09 *$ & $3.15 \pm 0.37$ \\
\hline & $5: 1$ & $83.53 \pm 8.3^{*}$ & $0.24 \pm 0.031$ & $-17.93 \pm 2.29$ & $28.90 \pm 3.45^{*}$ & $4.82 \pm 0.58$ \\
\hline & $2.5: 1$ & $178.35 \pm 44.34$ & $0.448 \pm 0.240$ & $-25 \pm 7.35$ & $19.44 \pm 3.56$ & $5.55 \pm 1.02$ \\
\hline \multirow[t]{3}{*}{ NOS-P5 } & $10: 1$ & $137.07 \pm 7.50 *$ & $0.420 \pm 0.036$ & $-9.43 \pm 7.61$ & $0.81 \pm 0.2$ & $0.07 \pm 0.02$ \\
\hline & $5: 1$ & $128.95 \pm 1.77^{*}$ & $0.35 \pm 0.037$ & $-8.16 \pm 5.15$ & $5.65 \pm 0.07$ & $0.94 \pm 0.01$ \\
\hline & $2.5: 1$ & $154.90 \pm 6.69$ & $0.37 \pm 0.026$ & $-12.95 \pm 0.49$ & $7.11 \pm 6.06$ & $2.03 \pm 1.73$ \\
\hline \multirow[t]{3}{*}{ NOS-P6 } & $10: 1$ & $782.40 \pm 64.21 *$ & $0.74 \pm 0.050$ & $-6.83 \pm 1.20$ & $91.96 \pm 1.54 *$ & $8.36 \pm 0.14$ \\
\hline & $5: 1$ & $876.75 \pm 18.17 *$ & $0.79 \pm 0.018$ & $-5.82 \pm 0.49$ & $65.71 \pm 3.15^{*}$ & $10.95 \pm 0.53$ \\
\hline & $2.5: 1$ & $306.27 \pm 48.01$ & $0.464 \pm 0.077$ & $-7.67 \pm 2.48$ & $2.28 \pm 0.74$ & $0.65 \pm 0.21$ \\
\hline
\end{tabular}

$* P<0.05$ compared with ratio $(2.5: 1)$

allowed to cool overnight to room temperature. The spectroflurometric measurement was done at excitation wavelength of $334 \mathrm{~nm}$, and emissions were recorded at wavelengths $I_{1}=369$ and $I_{3}=383 \mathrm{~nm}$ at slit widths for both excitation and emission at $3 \mathrm{~nm}$. Log values of polymer concentration were plotted versus $I_{1} / I_{3}$ to get CMC values.

Atomic Force Microscopy (AFM). Mica discs were fixed in metallic discs then cleaved many times with double-sided adhesive tape then used as substrates. NPs were prepared by nanoprecipitation in DI water then washed with the same vehicle twice by centrifugation to remove excess polymer. Samples were prepared by adding $10 \mu \mathrm{L}$ of NPs $(0.01 \mathrm{mg} /$ $\mathrm{mL}$ ) to freshly cleaved muscovite mica. After $30 \mathrm{~s}$, excess NPs dispersions were removed and samples were washed with $2 \times$ $200 \mu \mathrm{L}$ DI water. Samples were dried by exposing the mica discs to dry filtered air for $\sim 5$ min before being imaged in air (Atomic force microscope, Veeco Instruments, Santa Barbara, USA).

A Multimode AFM, equipped with E type scanner and NanoScope IIIa controller, was used throughout this study. The cantilevers used for imaging in air were silicon tapping probes (OMCL-AC160TS, Olympus) with a spring constant of 40-52 N/m operating at resonant frequencies of approximately 250-300 kHz. All imaging were conducted in tapping mode in air at $512 \times 512$ pixel resolution, with scan speeds of 0.5-1 Hz (41). Topographical images were plane-flattened and analyzed by the computer software Nanoscope Software v 7.3.

\section{In-Vitro Release of NOS from NPs}

A certain volume of NPs dispersion containing an amount equivalent to $100 \mu \mathrm{g}$ of NOS was diluted to a final volume of $2 \mathrm{~mL}$ in deionized water then transferred to a dialysis bag with a molecular weight cut-off $11 \mathrm{kDa}$. Release studies were done at two $\mathrm{pH}$ values in PBS buffer ( $\mathrm{pH} 7.4$ and $\mathrm{pH} 4.5)$, to simulate the $\mathrm{pH}$ of the environment faced by the NPs in the blood and the drop in $\mathrm{pH}$ to which NPs will be subjected in the tumor cells, respectively. Dialysis bags were kept in $25 \mathrm{~mL}$ of buffer within closed containers and shaken at $150 \mathrm{rpm}$ using a shaking water bath at $37 \pm 0.5^{\circ} \mathrm{C}$ (Thermostatically controlled shaking water bath, GFL, Germany) $(42,43)$. Samples of $0.5 \mathrm{~mL}$ were withdrawn from the release medium at 0.25 , $0.5,1,2,3,4,6$ and $8 \mathrm{~h}$ and replaced with fresh release medium. NOS concentration was then determined using high performance liquid chromatography (HPLC) (Agilent, USA). HPLC separation was done using the method specified by Chollet et al. at $35^{\circ} \mathrm{C}(44)$.

\section{Colloidal Stability Studies}

The colloidal stability of NPs was determined by measuring their resistance to sodium sulfate induced aggregation (45). $\mathrm{NP}_{\mathrm{s}}$ were prepared as described before and $1 \mathrm{~mL}$ of the prepared nanoparticle dispersions $(0.2 \% w / v$ in DI water) was added to $1 \mathrm{~mL}$ of sodium sulfate solution of different concentrations to reach the desired sodium sulfate molarity at a polymer concentration of $1 \mathrm{mg} / \mathrm{mL}$. The turbidity of the formed 
dispersions was measured spectrophotometrically (UV/VIS spectrophotometer, Jenway, UK) after $10 \mathrm{~min}$ at $500 \mathrm{~nm}$ and the critical coagulation concentration (CCG) was determined as the concentration at which a significant increase in turbidity was noticed using a plot of sodium sulfate concentration versus turbidity value.

\section{Cytotoxic Activity Assays}

The cytotoxicities of NOS solution, NOS-P1 and NOS-P4 NPs (Table 2) were determined using the sulphorhodamineB (SRB) method and monolayers of MCF-7 human breast cancer cells $(46,47)$. The cancer cells were grown in RPMI1640 medium, containing 10\% heat inactivated FBS, 50 units $/ \mathrm{mL}$ of penicillin and $50 \mathrm{mg} / \mathrm{mL}$ of streptomycin. Cells were maintained at $37^{\circ} \mathrm{C}$ in a humidified atmosphere containing $5 \% \mathrm{CO}_{2}$. Serial subculturing was used to maintain the cells as a "monolayer cultures".

Cells were collected by $0.25 \%$ Trypsin-EDTA and seeded in 96-well plates at a density of $1-2 \times 10^{3}$ cells/well in a medium supplemented with RPMI-1640. After $24 \mathrm{~h}$, various concentrations $(0.1-1000 \mu \mathrm{mol} / \mathrm{l}$ of NOS and equivalent amounts of NOS in NPs) were added to each well and cells were incubated for $72 \mathrm{~h}$. After $72 \mathrm{~h}$ treatment, 10\% trichloroacetic acid was added and left for $1 \mathrm{~h}$ at $4^{\circ} \mathrm{C}$ for fixation of cells followed by staining of the treated wells for $10 \mathrm{~min}$ at room temperature using $0.4 \% \mathrm{SRB}$ dissolved in $1 \%$ acetic acid. The plates were air dried for $24 \mathrm{~h}$ then Tris-HCl was added and the plates were left shaken at $1600 \mathrm{rpm}$ for $5 \mathrm{~min}$ to solubilize the dye. The optical density (OD) of each well was measured spectrophotometrically at $564 \mathrm{~nm}$ using an ELISA microplate reader (ELISA microplate reader, ChroMate$4300, \mathrm{FL}, \mathrm{USA})$. The inhibitory concentration of $50 \%\left(\mathrm{IC}_{50}\right)$ values were determined according to the equation for Boltzman sigmoidal concentration-response curve using the nonlinear regression fitting models (Graph Pad, Prism Version 5).

\section{Pharmacokinetic Studies on NOS Loaded NPs}

The protocol for these studies was reviewed and approved by the research ethics committee of Faculty of Pharmacy, Ain Shams University, Cairo, Egypt. All glasswares used for preparing solutions and NPs were sterilized by autoclaving, and the entire preparation procedures were carried out in a laminar flow hood under aseptic conditions. Ninety female Wistar albino rats (120-160 g) were divided into three groups, each comprising 30 rats. The different groups were as follow: Group 1 received i.v. NOS- P1 NPs, Group 2 received i.v. NOS- P4 NPs, Group 3 received i.v. NOS solution (in dilute HCL) (48). NOS and the equivalent amount of NOS-NPs were administered for all animals in a dose of $50 \mathrm{mg} / \mathrm{kg}$ in a volume of $1 \mathrm{~mL}$. Intravenous administration was done through the peripheral tail vein of the rat. At different time intervals $0.083,0.167,0.25,0.5,1,2,4,6,8$, and $12 \mathrm{~h}$ following administration of NOS or NPs, blood samples were collected from 3 rats through the retro-orbital vein using heparinized Helmington microcapillary tubes into polypropylene microcentrifuge tubes containing $25 \mu \mathrm{L}$ of $20 \mathrm{mM}$ sodium citrate, centrifuged at $4600 \mathrm{rpm}$ for $15 \mathrm{~min}$ and the separated plasma samples were stored at $-80^{\circ} \mathrm{C}$ until analyzed (42). Acetonitrile was added to the thawed plasma samples for protein precipitation and extraction of NOS (49).

The mixture was vortexed for $5 \mathrm{~min}$ (Vortex mixer, IKA MS3 Digital, Wilmington, Germany) to extract both free NOS and NP bound, then centrifuged at $3000 \mathrm{rpm}$ for $10 \mathrm{~min}$ (cooling centrifuge, Hermle, Germany), and finally filtered through a $0.45 \mu \mathrm{m}$ membrane filter. Samples of $50 \mu \mathrm{l}$ were injected into the HPLC apparatus for analysis (Agilent HPLC, USA). HPLC assay was performed by modification of the method specified by Chollet et al. at $40^{\circ} \mathrm{C}$ using papaverine as internal standard (44).

Plasma concentration data were analyzed using PKSolver Excel Add-in (50). The mean (free and NP bound) NOS concentrations in plasma were plotted against time. Then the following pharmacokinetic parameters were assessed: The maximum plasma concentration $\mathrm{C}_{\max }(\mathrm{ng} / \mathrm{mL})$, the half-life $\mathrm{t}_{1 / 2}(\mathrm{~h})$, the area under NOS concentration-time curve up to the last sampling point $\mathrm{AUC}_{0-12}(\mathrm{ng} / \mathrm{mL} \mathrm{h})$, the area under NOS concentration-time curve with extrapolation to infenity $\mathrm{AUC}_{\mathrm{inf}}(\mathrm{ng} / \mathrm{mL} \mathrm{h})$, and the mean residence time $\mathrm{MRT}(\mathrm{h})$. The area under the curve was calculated by the linear trapezoidal rule up to the last sampling point $\left(\mathrm{AUC}_{0-12}\right)$ with extrapolation to infinity ( $\left.\mathrm{AUC}_{\mathrm{inf}}\right)$.

\section{Toxicity Studies}

Determination of maximum tolerated dose and the lethal dose $50 \%\left(\mathrm{LD}_{50}\right)$ of NOS solution and NOS- P1 NPs was carried out as specified by Moura et al. (51). Twenty female Wister rats in groups of 5 were intravenously injected via the tail vein following a dose-escalation schedule based on three dose levels of NOS as solution and NOS- P1 NPs (50, 100, and $200 \mathrm{mg}$ $\mathrm{NOS} / \mathrm{kg}$ as a single-dose) and saline was administered to a group of 5 rats as a control. These concentrations were selected as NOS is well known to have $\mathrm{LD}_{50}$ in mice of 83 $\pm 6.1 \mathrm{mg} / \mathrm{kg}$ (48). Animals were observed until day 7 for any death occurrence and body weights were measured every 2 days to determine the maximum tolerated dose (defined as the dose that causes approximately 15\% loss in body weight and does not result in lethality) and the lethal dose of $50 \%$ $\left(\mathrm{LD}_{50}\right)$ that is defined as the administered dose which causes death of $50 \%$ of the animals, determined by interpolation of results (death percentage in the group/dose). 


\section{Hematological Toxicity}

Twenty-four hours after the injection of a single $200 \mathrm{mg} / \mathrm{kg}$ dose of NOS as solution, NOS-P1 NPs and saline into female Wistar rats, blood samples were withdrawn by retro-orbital sinus puncture using heparinized Helmington microcapillary tubes. Hematological toxicity was determined from the data of hematology parameters which include erythrocytes count, platelets, leukocytes and leukocytes differential count by using blood cell counter (Cell Dyn 1700, Abbott, USA) (52). Blood smears were prepared as soon as possible after blood collection on a glass slide and quickly dried and stained with Leishman's stain for the differential blood count.

\section{Histopathological Analysis}

At the end of the observation period (7 days) of treatment of the rats with NOS, NOS-P1 NPs (Toxicity studies, 2.2.8) and the saline control groups, selective organs (liver, kidney, spleen, brain, heart and lung) were dissected out. Organs were formalin-fixed and embedded in paraffin then sectioned into $5 \mu \mathrm{m}$ sections and stained with Hematoxylin and Eosin (H\&E). Microscopic evaluation was done by a pathologist (Light microscope, Carl Zeiss Axiostar Plus, Göttingen, Germany).

\section{Statistical Analysis}

All data were expressed as mean \pm standard deviation (SD). Comparison of the mean values was undertaken using ANOVA test followed by Tukey post-Hoc test for multiple comparisons with SPSS statistical software program, USA. $p<0.05$ were considered statistically significant.

\section{RESULTS AND DISCUSSION}

\section{Characterization of Block Copolymers}

Block copolymers were efficiently prepared by ring opening polymerization of different molecular weights of PCL blocks using mPEG $1.9 \mathrm{kDa}$ and PEG $2.0 \mathrm{kDa}$ as starting materials respectively. ${ }^{1} \mathrm{H}-\mathrm{NMR}$ spectra (Figure $\mathrm{S} 1$ ) of the prepared block co-polymers were produced, which showed the characteristic peaks of PCL at $1.40 \mathrm{ppm}(\beta$-methine $(\mathrm{CH})$ proton, quintet split; Figures S1-S2 (d)), $1.64 \mathrm{ppm}(\gamma$ and $\delta$-methine $(\mathrm{CH})$ proton, quintet split; Figures S1-S2 (c,b)), $2.28 \mathrm{ppm}(\alpha-$ methine $(\mathrm{CH})$ proton, triplet split; Figures S1-S2 (e)) and 4.04 ppm ( $\varepsilon$-methine $(\mathrm{CH})$ proton, triplet split; Figures $\mathrm{S} 1-$ S2 (a)) and the characteristics peak of PEG at $3.65 \mathrm{ppm}$ (methylene $\left(\mathrm{CH}_{2}\right)$ protons, triplet split; Figures S1-S2 (f)). The molecular weights of the block co-polymers (Table 1) were calculated using integration of ${ }^{1} \mathrm{H}-\mathrm{NMR}$ chromatograms as well as
GPC. The CMC of the P1-6 micelles was determined by a florescence technique using pyrene as a probe (Table 1). It can be observed that the CMC values of the polymers were in the range $(0.48-7.08 \mathrm{mg} / \mathrm{L})$ and it can be noticed that for either di- or triblock copolymers, CMC values decreased as the molecular weight of the hydrophobic part (PCL) increased. Unexpectedly, P5 and P6 showed low values of CMC when compared to other polymers with higher hydrophilicity $(\mathrm{P} 1)$. Although P5 and P6 had high hydrophilicity as indicated by

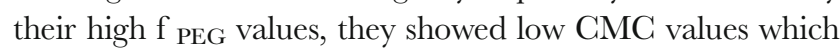
may be due to the higher density of PCL blocks per molecule (4 and 6 respectively) which render the aggregation process much easier. This would suggest that the star shaped polymers due to their architecture can produce more stable micelles that can resist dilution after injection compared to linear polymer (53).

\section{Characterization of Prepared NPs}

\section{Particle Size, Zeta Potential and Entrapment Efficiency Determinations}

Table 2 shows the particle size of NPs produced from different block co-polymers with different polymer to drug ratios as well as their zeta potentials and entrapment efficiencies. Particle sizes were in the range from 65.61 to $876.75 \mathrm{~nm}$, zeta potentials were in the range from -5.82 to $-25 \mathrm{mV}$ and entrapment efficiencies were in the range from 2.28 to $91.96 \%$. Particle size was used to select the most suitable NPs for further investigations. P5 and P6 were excluded from selection as their NPs showed either a very low entrapment efficiency (NOS-P5) or a large particle size (NOS-P6) and both are not suitable for cancer drug delivery (37). Polymer configuration of P6 may be the main determinant for NP stability and size as the polymer will be more restricted to localized spots on the NP surface rather than covering the whole NP surface (Fig. 1) allowing non-shielded areas to interact and form large sized NP aggregate. Statistical analysis showed that in most NPs the ratio 2.5:1 had particle sizes that were significantly higher than that of the ratios 5:1 and 10:1 $(p<0.05)$. Meanwhile, there were no significant differences between the particle sizes of NPs obtained from ratios 5:1 and 10:1. The ratio 5:1 was selected as the preferred ratio since it in general produced smaller particles than the 2.5:1 ratio and a particle size equivalent to those produced using the ratio 10:1 with a lower polymer amount. This together with steric stabilization and hydrophilicity contributes to their decreased uptake by the RES (9,54-56).

Regarding the zeta potential, all tested NPs were found to have negative values that ranged from -5.82 to $-25 \mathrm{mV}$. NOS-P1 and P4 showed significantly $(p<0.05)$ higher charge values when compared to NPs with the same polymer to drug ratios, however, NOS-P5 and P6 showed the lowest values of 
zeta potential. Although $\mathrm{P} 5$ and $\mathrm{P} 6$ have high $\mathrm{f}_{\mathrm{PEG}}$ values compared to $\mathrm{P} 2-\mathrm{P} 4$ and hence lower surface charge $(-5$ to $-12 \mathrm{mV}$ ). With exception of NOS-P1, it can be noticed that particles prepared using polymers with high $\mathrm{f}_{\mathrm{PEG}}$ have surfaces with low negativity (NOS-P3, 5 and 6), however those with low $f_{\mathrm{PEG}}$ have surfaces are more negative (NOS-P4) (57).

Due to their small size and considerable entrapment efficiency at polymer to drug ratio (5:1), NOS containing NPs (NOS-P1 and NOS-P4) were selected for further investigations as their size and PEG coated surfaces made them good candidates as delivery systems for cancer treatment.

\section{Atomic Force Microscopy (AFM)}

From the data above two different NOS loaded nanoparticle systems (NOS-P1 and NOS-P4) at polymer to drug ratio $(5: 1)$ were selected for further investigation. Figure 2 shows the AFM images of these NOS loaded NPs (NOS-P1 and NOSP4) with recorded particle diameters of 165.6 and $132.13 \mathrm{~nm}$ and heights of 44.9 and $18.79 \mathrm{~nm}$, respectively, as determined by section analysis (Fig. 2). The sizes obtained by AFM were found to be larger than those obtained by DLS. This could be due to the nature of the micellar like particles, which are soft and easily deform under the force applied during AFM imaging. It was described by Roos et al., that nanoparticles can suffer from nanoindentation followed by particle expansion during AFM imaging (58), when imaged using the tapping mode, thereby being suppressed with the highly resonating tips of the device against the mica substrate resulting in an increase in particle size and decrease in particle height. This was especially the case when using cantilevers with a high spring constant value $(\mathrm{K}=40-50 \mathrm{~N} / \mathrm{m})$. It is worth to say that in the present study, it was not possible to use the contact mode for AFM imaging since the NPs were too soft and adhered and changed the shape of the imaging AFM tips while scanning the samples, thus producing very low quality images.
Zhang et al. using DSC measurement showed that similar copolymers exhibited low glass transition temperatures suggesting that nanoparticles formed with these materials would be soft and deformable at body temperature. Hence these nanoparticles would be more capable of surviving splenic filtration and passing through cancer tissue fenestration than rigid ones (59).

\section{In-Vitro Release of NOS from NPs}

Figure 3 shows the release patterns of NOS from the NPs (NOS-P1 and NOS-P4) with polymer to drug ratio (5:1) at $\mathrm{pH}$ values of 7.4 and 4.5. It was found that NOS release was significantly higher and faster (after $15 \mathrm{~min}$ ) at $\mathrm{pH} 4.5$ when compared to $\mathrm{pH} 7.4(p<0.05)$. A similar behavior was previously reported for release of NOS and other basic drugs from polymeric nanoparticles when tested at acidic conditions $(34,42,60)$. It is to be noted that after $1 \mathrm{~h}$ the more hydrophilic NOS-P1 showed significantly higher release rates compared to that of NOS-P4 prepared using polymer with low $f_{\mathrm{PEG}}$ value. After 8 h, NPs at pH 7.4 released only 25.44 and $36.3 \%$ of their NOS load, while at $\mathrm{pH} 4.5,85.7$ and $99.7 \%$ of the drug from NOS-P4 and NOS-P1 respectively. The high release rates of NOS in acidic $\mathrm{pH}$ is expected because of its basic nature which suggest high drug solubility at low $\mathrm{pH}$ values and hence higher release rates from polymeric NPs (34,60,61). This drug release characteristics will allow the NPs to continue carrying the bulk of NOS while circulating in the blood and releasing their load majorly in the tumor tissue due to its lower $\mathrm{pH}$ (62). This should lead to a significant increase in therapeutic effect of NOS and prevention of drug losses if widely distributed within the body.

It was also observed from Fig. 3 that NOS-P1 showed the highest release profile compared to NOS-P4 at either $\mathrm{pH}$ values. This may be attributed to the low molecular weight of polymer P1 compared to P4, combined with P1 being composed of only two polymer blocks that hence would be
Fig. 2 AFM image of nanoparticles formed from: (a) NOS-PI at polymer to drug ratio (5: I). (b) NOS-P4 at polymer to drug ratio (5:I).
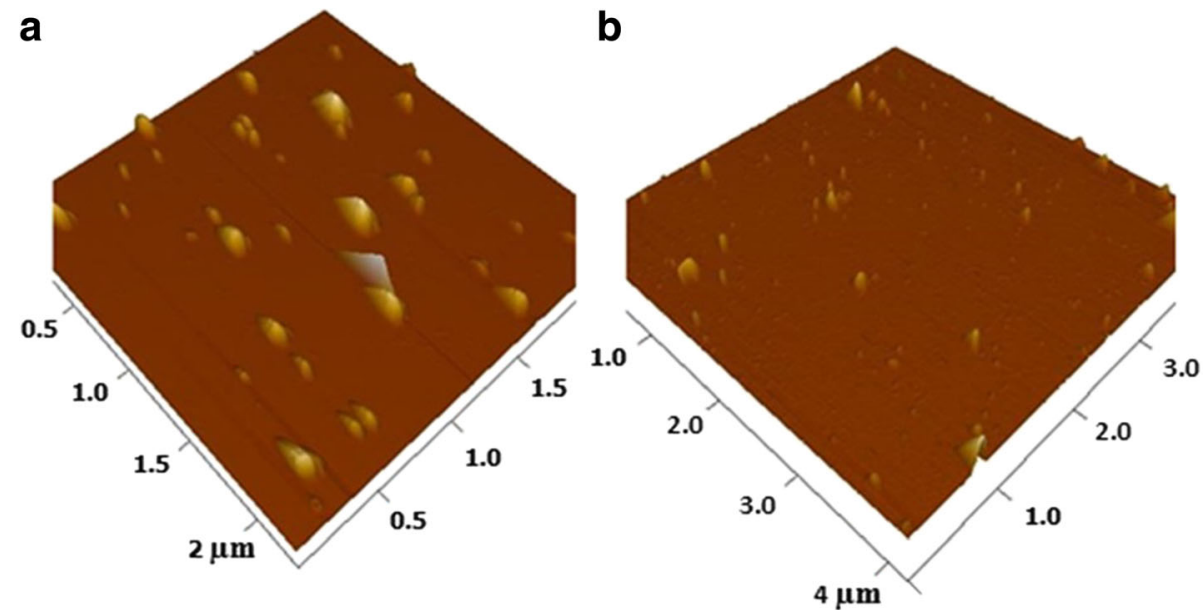


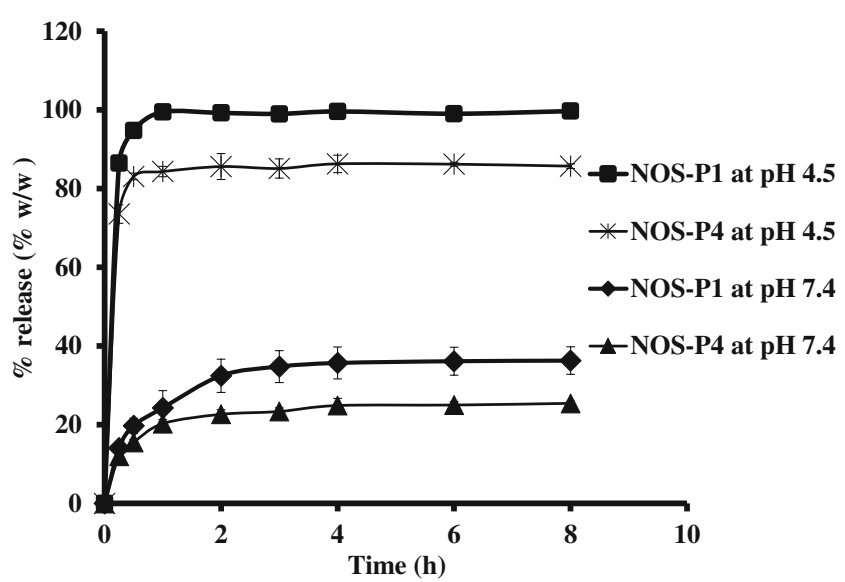

Fig. 3 In-vitro release of NOS from nanoparticles (NOS-PI and NOS-P4) with polymer to drug ratio (5:I) at $\mathrm{pH} 7.4$ and 4.5 .

expected to form only weak Van der Waals interaction with NOS. Accordingly, NOS more easily escaped from the less dense matrix of NOS-P1 NPs as compared to that of the other NP formulation composed of a block-copolymer of higher molecular weight (triblock co-polymer chain) and hydrophobicity (Table 1).

\section{Colloidal Stability Studies}

As shown in Fig. 4, the selected types of NPs showed different degrees of stability when mixed with increasing concentrations of sodium sulfate. NOS-P1 showed the highest critical coagulation concentration $(\mathrm{CCC}=0.5 \mathrm{M})$ indicating its high stability against electrolytes. NOS-P4 NPs showed a reasonable, but less stable behavior against increasing sodium sulfate concentration, (i.e. $\mathrm{CCC}=0.3 \mathrm{M}$ ). This can be explained by the degree of effective hydration of the NPs by the water sheath and steric stabilization, which depends on the amount, length and freedom of movement of the PEG on the surface of the

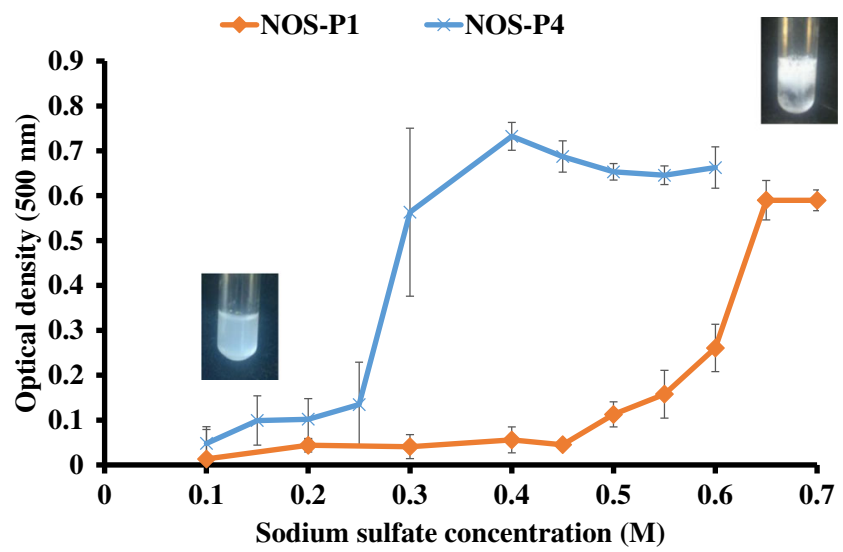

Fig. 4 Determination of the critical coagulation concentration (CCC) of NOS containing nanoparticles (NOS-PI and NOS-P4) with polymer to drug ratio $(5: 1)$ assessed by the turbidity method. particles. P1 comprises the greatest amount of PEG $\left(\mathrm{f}_{\mathrm{PEG}}=0.63\right)$ compared to $\mathrm{P} 4\left(\mathrm{f}_{\mathrm{PEG}}=0.26\right)($ Table 1$)$.

Although NOS-P1 and P4 NPs showed no significant difference in measured zeta potential at all tested polymer to drug ratios the difference in measured CCG values could be interpreted based on polymer conformation on the NP surface. $\mathrm{P} 1$ is a di-block-copolymer consisting of a hydrophilic PEG chain attached to a single PCL block and hence PEG will be freely protruding on the surface of the micellar type nanoparticle. Such a conformation would be more stable to the charge neutralizing effect of sodium sulfate than particles made of tri-block-copolymers, where the PEG is attached in both ends to PCL blocks (P4) and would be expected to form flower like NPs with PEG in loop confirmation on its surface and with less freedom of movement (63). The flower like conformation of PEG chains would allow the exposure of more hydrophobic PCL areas that would easier allow the process of NOS- P4 NPs aggregation.

Hence, the NPs produced from the $\mathrm{P} 1$ polymer express the highest colloidal stability which results suggest that the NPs tested here will not aggregate in blood, which contribute to their safety when clinically administered.

\section{Cell Cytotoxicity}

The cytotoxicity profiles of NOS solution, NOS-P1 and NOS-P4 NPs were examined using the SRB assay to evaluate their effect on MCF-7 cell line survival (Fig. 5). Cytotoxicity analysis indicated that the $\mathrm{IC}_{50}$ value of NOS-P1 NPs was equivalent to $6.916 \mu \mathrm{mol} / \mathrm{l}$ respectively, which were significantly lower than that of NOS-P4 NPs $(38.92 \mu \mathrm{mol} / \mathrm{l})$, as well as NOS solution $(41.11 \mu \mathrm{mol} / \mathrm{l})(P<0.05)$. The difference in cytotoxicity may be due to the higher release of NOS from NOS-P1 NPs compared to NOS-P4 NPs when in an acidic medium.

A cytotoxicity assay for polymers ( $\mathrm{P} 1$ and $\mathrm{P} 4)$ was also done and we found that the polymers had little or no cytotoxic effect

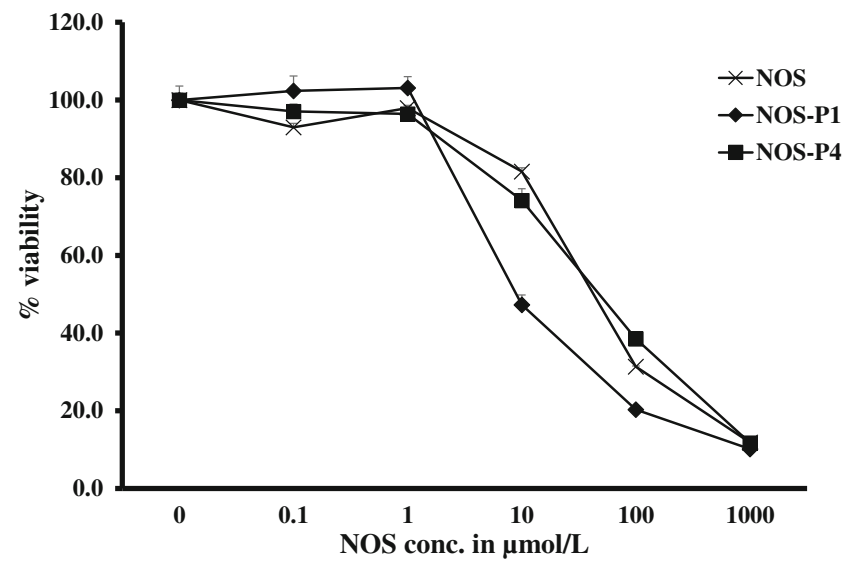

Fig. 5 Cytotoxicity of NOS solution, NOS-PI and NOS-P4 to MCF-7 cells. 
on the cells (data not shown). The above mentioned results indicate that the increased cytotoxic effect of the NOS NPs compared to NOS solution could be due to a combination of the small particle size of the NPs resulting in increased uptake into the cells and the selective increased acidic $\mathrm{pH}$ release of NOS-P1.

\section{Pharmacokinetic Studies on NOS and Selected NOS-NPs}

Figure 6 shows the mean NOS concentrations in plasma in rats after i.v. administration of NOS-P1, NOS-P4 NPs (polymer to drug ratio (5:1)) and NOS solution and the corresponding pharmacokinetic parameters are shown in Table 3. It should be noted that since the HPLC method extracts NOS from the plasma samples, not only free NOS but also NOS from the NP will be extracted, and hence the plasma concentrations given are a combination of free and NP bound NOS.

It was found that the NOS solution showed the highest plasma concentration (Cmax) compared to both NPs investigated (NOS-P1 and NOS-P4). Both NPs released NOS in the blood at low concentrations as supported by the in-vitro release test at pH 7.4 (Fig. 3). It should also be noted that both NP systems showed an initial high plasma concentration of NOS followed by an abrupt decrease. This may be due to desorption of NOS from the surface of NPs followed by rapid distribution and uptake of the drug into different organs as previously described by other research groups $(13,64)$.

The biodistribution of NPs in different organs of the body, especially the liver and spleen, after IV injection, is mainly controlled by interaction with the RES and can to some degree be modified by the size and the surface characteristics, such as hydrophilicity and steric stabilization, of the nanoparticles. The more hydrophobic the NPs are and the lower the amount of PEG that exists on their surfaces, the faster they become opsonized and removed by the RES system. On the contrary, high coverage of NPs surfaces with PEG and a high degree of steric stabilization decreases their opsonization and uptake by the RES system $(42,65)$. The selected NPs have the value of $f_{P E G}$ in the following order NOS-P1>NOSP4 (Table 1). Furthermore, as discussed above the NOS-P1 NPs are expected to show a high degree of steric stabilization with a resultant low RES capture and a long blood circulation time. Moreover, NOS as a hydrophobic drug can be adsorbed to the hydrophobic moieties of the copolymer in the NPs. NOS-P4 showed the highest initial concentration of NOS at 5 min $(P<0.05)$, compared to NOS-P1, which may be attributed to the moderate amount of PEG on the surfaces which would allow most of the hydrophobic drug, NOS, to be adsorbed on the surface in-between the PEG chains. When the NPs entered the blood stream the drug molecules desorbed in the plasma resulting in a high initial concentration, followed by a sudden drop in plasma levels due to opsonization and uptake by the RES system. NOS-P1 NPs, comprising the highest amount of PEG (Table 1), showed a moderate level of NOS concentration in plasma which was maintained for longer time than the NOS solution and the NOS-P4. It was also noticed, that the NOS solution showed a biphasic elimination profile as previously reported by Gibaldi and Weiner (66). They hypothesized that NOS is rapidly eliminated from plasma compartment while being stored in adipose tissue from which it is eliminated at a lower rate imparting NOS its biphasic elimination. Upon observation of NOS NPs, it was found that the hydrophilic property of NPs reduced their deposition into adipose tissue, which resulted in reduction of the biphasic elimination of NOS as shown in Fig. 6.

These results were supported by the pharmacokinetic parameters shown in Table 3. MRT and $t_{1 / 2}$ of NOS-P1 NPs was significantly higher than that of NOS solution $(p<0.05)$. $\mathrm{AUC}_{0-12}$ and $\mathrm{AUC}_{0-\mathrm{inf}}$ are indicators of availability of NOS in the injected delivery system. NOS solution showed higher $\mathrm{AUC}_{0-12}$ than NOS-P4 NPs but not higher than that of NOSP1 NPs and this finding was expected from the soluble form of
Fig. 6 Mean NOS concentrationtime profiles in the plasma of rats after i.v. injection of nanoparticles (NOS-PI and NOS-P4) with polymer to drug ratio (5:I) and NOS solution.

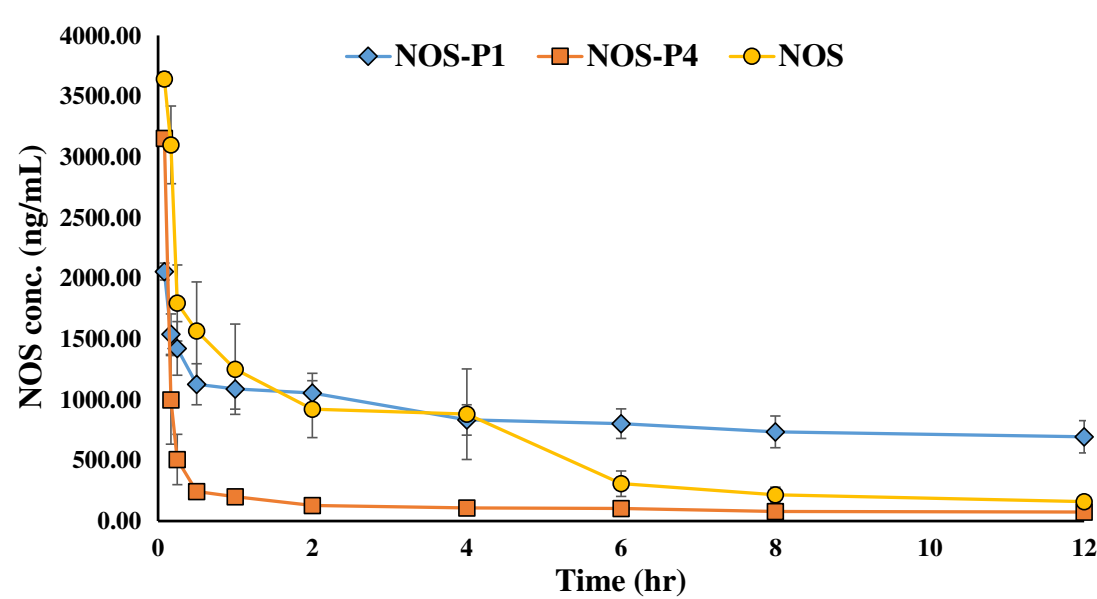


Table 3 Pharmacokinetic Parameters of NOS in Rat Plasma After i.v. Injection of Nanoparticles (NOS-PI and NOS-P4) with Polymer to Drug Ratio (5:I) and NOS Solution

\begin{tabular}{lccc}
\hline Parameter & Mean \pm SD & \\
\cline { 2 - 4 } & Group I (NOS-PI) & Group 2 (NOS-P4) & Group 3 (NOS) \\
\hline$C_{\max }(\mathrm{ng} / \mathrm{mL})$ & $2055.97 \pm 98.89 *$ & $3152.49 \pm 84.99 *$ & $3642.28 \pm 100.71$ \\
$\mathrm{~T}_{1 / 2}(\mathrm{hr})$ & $22.92 \pm 9.2 *$ & $15.98 \pm 5.21$ & $5.12 \pm 3.02$ \\
MRT $(\mathrm{hr})$ & $32.4 \pm 12.93 *$ & $15.52 \pm 3.65$ & $5.31 \pm 0.42$ \\
AUC $_{0-12}\left(\mathrm{ng} / \mathrm{mL}^{*} \mathrm{hr}\right)$ & $9351.74 \pm 1970.57$ & $2358.67 \pm 759.85 *$ & $7308.96 \pm 2116.77$ \\
AUC $_{\text {0-inf }}\left(\mathrm{ng} / \mathrm{mL}^{*} \mathrm{hr}\right)$ & $28365.57 \pm 2799.95^{*}$ & $3959.34 \pm 1520.79$ & $8317.39 \pm 2300.66$ \\
\hline
\end{tabular}

Cmax: the maximum plasma concentration $(\mathrm{ng} / \mathrm{mL}), \mathrm{tl} / 2$ : the half-life $(\mathrm{h}), \mathrm{AUCO}-\mathrm{I2}$ : the area under NOS concentration-time curve up to the last sampling point ( $\mathrm{ng} / \mathrm{mL}$ h), AUCO-inf: the area under NOS concentration-time curve with extrapolation to infinity $(\mathrm{ng} / \mathrm{mL} h)$, and MRT: the mean residence time $(h)$

* $P<0.05$ compared with NOS solution the drug. On the contrary, NOS-P1 had the highest $\mathrm{AUC}_{0 \text {-inf }}$ compared to NOS solution and NOS-P4. This is thought to be due to the higher density of PEG chains on the surface of NOS-P1 which imparted a stealth property and for them resulting in longer half-life and longer blood circulating property $(67,68)$.

The present results indicate that the incorporation of NOS in NOS-P1 NPs resulted in an increased half-life and hence availability of NOS to cancer tissue. The increase in NOS concentration in the blood after administration of NOS-P1 compared to NOS control solution may exceed the bioavailability of NOS injection in the soluble form and increase the duration and effect of the injected dose of NOS.

\section{Toxicity Studies}

When comparing the different tested nanoparticle formulations, NOS-P1 NPs showed the highest in-vitro drug release profile, the longest circulation time and the lowest $\mathrm{IC}_{50}$ of the selected NPs. Hence, NOS-P1 NPs was selected for further investigation of in vivo toxicity to various tissues in rats. Upon observing the rats for 7 days, there were no deaths recorded among the rats used in this experiment during the test period after the administration of 50,100 and $200 \mathrm{mg} / \mathrm{kg}$ of NOS and the equivalent concentrations of NOS-P1 in nanoparticles. In addition, no significant weight loss $(P<0.05)$ exceeding $15 \%$ of the initial body weight was observed in the rats with both drug solution and nanoparticles (Fig. 7a and b). Hence, it was not possible to determine the lethal dose or the maximum tolerated dose from this experiment. Higher doses of NOS could only be administered by increasing the solution volume to non-injectable values.

The cytotoxicity assay showed that NOS-P1 NPs was able to kill cancer cells at a much lower concentration than a NOS solution. In the simple toxicity study, it was further found that NOS-P1 was safe for normal cells of the body. This test indicated the safety of using these NPs clinically for cancer treatment especially if efficiently targeted to cancer tissues.

\section{Hematological Toxicity}

The results of our previous toxicity studies were supported by testing the hematological toxicity of NOS solution and NOSP1 NPs at $200 \mathrm{mg} / \mathrm{Kg}$ NOS in rats. After measuring hematology parameters from rats injected with NOS, NOS-P1 NPs and saline, there was no significant difference $(p<0.05)$ observed in the blood cell counts between different groups as shown in Fig. 8. The results of this test together with that of
Fig. 7 Weights of rats after injection with NOS solution (a), NOS-PI nanoparticles $(\mathbf{b})$ at concentrations of 50, 100 and $200 \mathrm{mg} / \mathrm{kg}$ compared to negative control (saline) and observation for 7 days $(n=5)$.
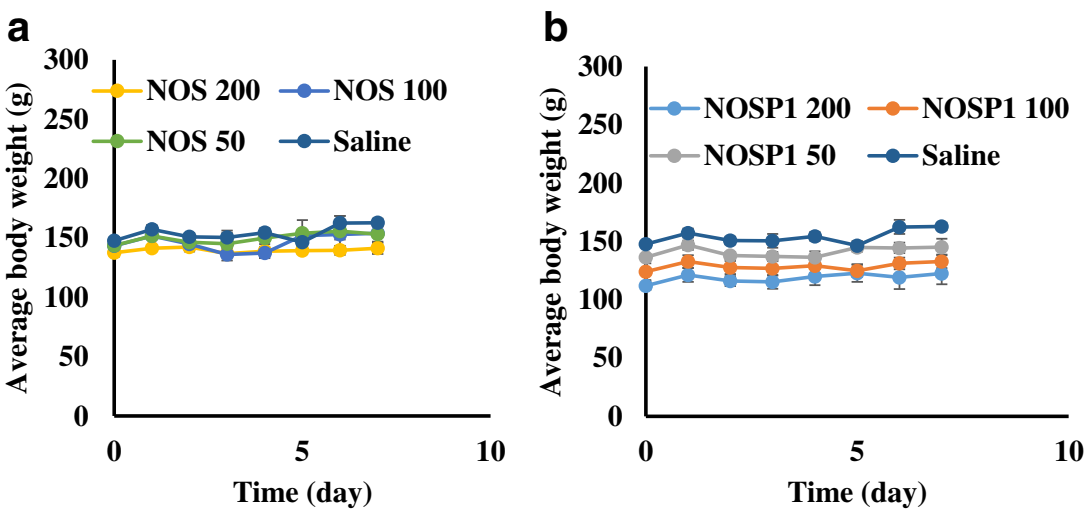
a

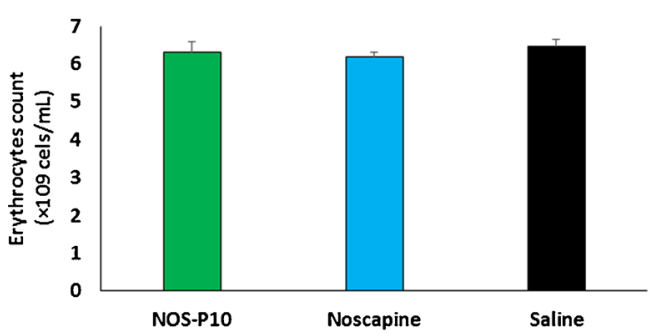

C

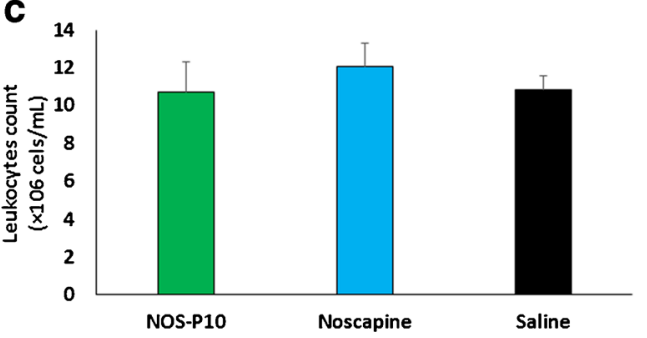

b

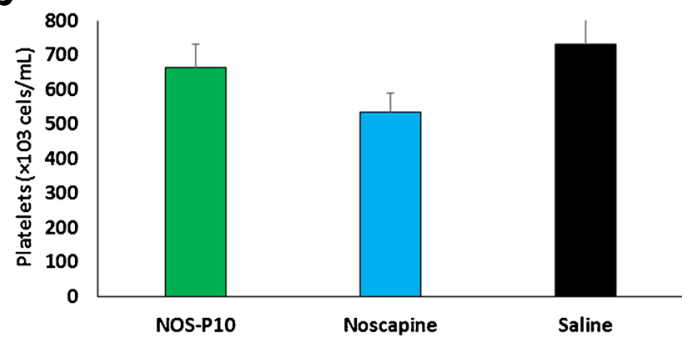

d

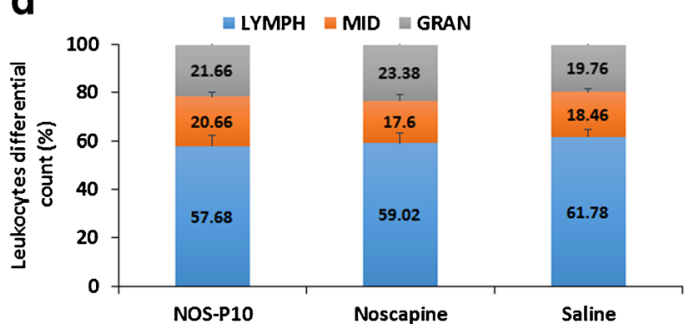

Fig. 8 Haematological toxicity of NOS solution and NOS-PI nanoparticles. (a) erythrocytes count, (b) Platelets count, (c) Leucocytes count, and (d) Differential leucocytes count. LYMPH: lymphocytes, MID: the mid-cell fraction (eosinophils, basophils, monocytes, and precursors of WBCs), GRAN: granulocytes $(p<0.005)$.

colloidal stability study gives us a good indication about the safety of using of these formulation in clinical trials. In a similar study using doxorubicin loaded HA-PEG-PCL NPs, it was found that incorporating the anticancer drug in the NPs improved its haemocompatability (69).

\section{Histopathological Analysis}

The histopathological analysis showed that a single treatment with NOS solution and NOS-P1 NPs (200 mg/kg NOS) did not result in any detectable pathological abnormalities in rats. Metastatic lesions were completely absent in the liver, brain, heart, kidneys, lungs and spleen in both NOS solution and NOS-P1 NPs treated groups (Fig. 9).

\section{CONCLUSIONS}

A series of PEG/PCL block copolymers were synthesized using ring opening polymerization and used to formulate nanozised drug delivery systems.

Combining data of zeta potential and particle sizes, we found that increasing the number of polymer PEG blocks in the polymers (NOS-P5, NOS-P6) used for NP preparation resulted in a decrease in surface charge but was associated with increased NP size and the probability of NP aggregation. The NP with the highest $\mathrm{f}_{\mathrm{PEG}}$ produced from the linear PCLPEG was shown to have the smallest particle size and a high degree of stability to high concentrations of sodium sulfate. The NPs produced using the polymer to drug ratio (10:1 and 5:1) were of a size significantly smaller than polymer to drug ratio (2.5:1). As there was no significant difference between the sizes of NPs produced at polymer to drug ratio 10:1 and 5:1, we decided to use the ratio with the least amount of polymer. Among tested polymers, $\mathrm{P} 1$ and $\mathrm{P} 4$ were selected for further investigations due to the small size of NOS loaded NPs when produced at polymer to drug ratio $(5: 1)(<100 \mathrm{~nm})$.

AFM imaging revealed that the particles were nearly spherical in shape and of small size. Release profiles of NOS loaded NPs (NOS-P1 and NOS-P4) with polymer to drug ratio (5:1 at $\mathrm{pH} 4.5)$ were significantly higher and faster than those obtained at $\mathrm{pH}$ 7.4. These results indicated that the NPs will continue carrying most of its content of NOS while circulating in the blood, however they will release its load at the acidic extracellular environment of tumor tissue. Moreover, NOS-P1 NPs showed the highest extent in vitro drug release compared to NOS-P4. These results suggested a similar high stability of these NPs when injected to blood intravenously.

MCF-7 cell line cytotoxicity analysis showed that the formulation of NOS into NPs resulted in a dramatic increase in its cytotoxic activity most likely due to a combined effect of the small particle size and the selective NOS release (69-71). In addition, the NOS-P1 plasma profile was found to have a long half-life and MRT with a high values of $\mathrm{AUC}_{0-12}$ and $\mathrm{AUC}_{0-}$ inf indicating a higher availability for reaching potential tumor tissues.

On the other hand, injection of NOS solution and NPs into rats did not result in any deaths or weight loss among the groups of rats used. Therefore, it wasn't possible to determine either the maximum tolerated dose or the $\mathrm{LD}_{50}$. Moreover, toxicity of NOS solution and nanoparticle formulations on normal cells was evaluated by determination of their 
Fig. 9 Light photomicrograph showing H\&E- stained $10-\mu$ m-thick sections of liver $(\times 200)$, brain $(\times 200)$, heart $(\times 200)$, lung $(\times 200)$, kidney $(\times 200)$, spleen $(\times 100)$ from groups of rats $((\mathbf{a})$ Control, (b) NOS-PI nanoparticles and (c) NOS solution). No histopathological differences were noted in these tissues. hematological and histopathological toxicity. Hematology parameters and sections of different organ tissues showed no abnormality when compared to control group. So, it can be concluded that (at least in vitro) NOS loaded biodegradable NPs has increased NOS potency against cancer cells and in viwo enhanced its bioavailability and blood circulation time.
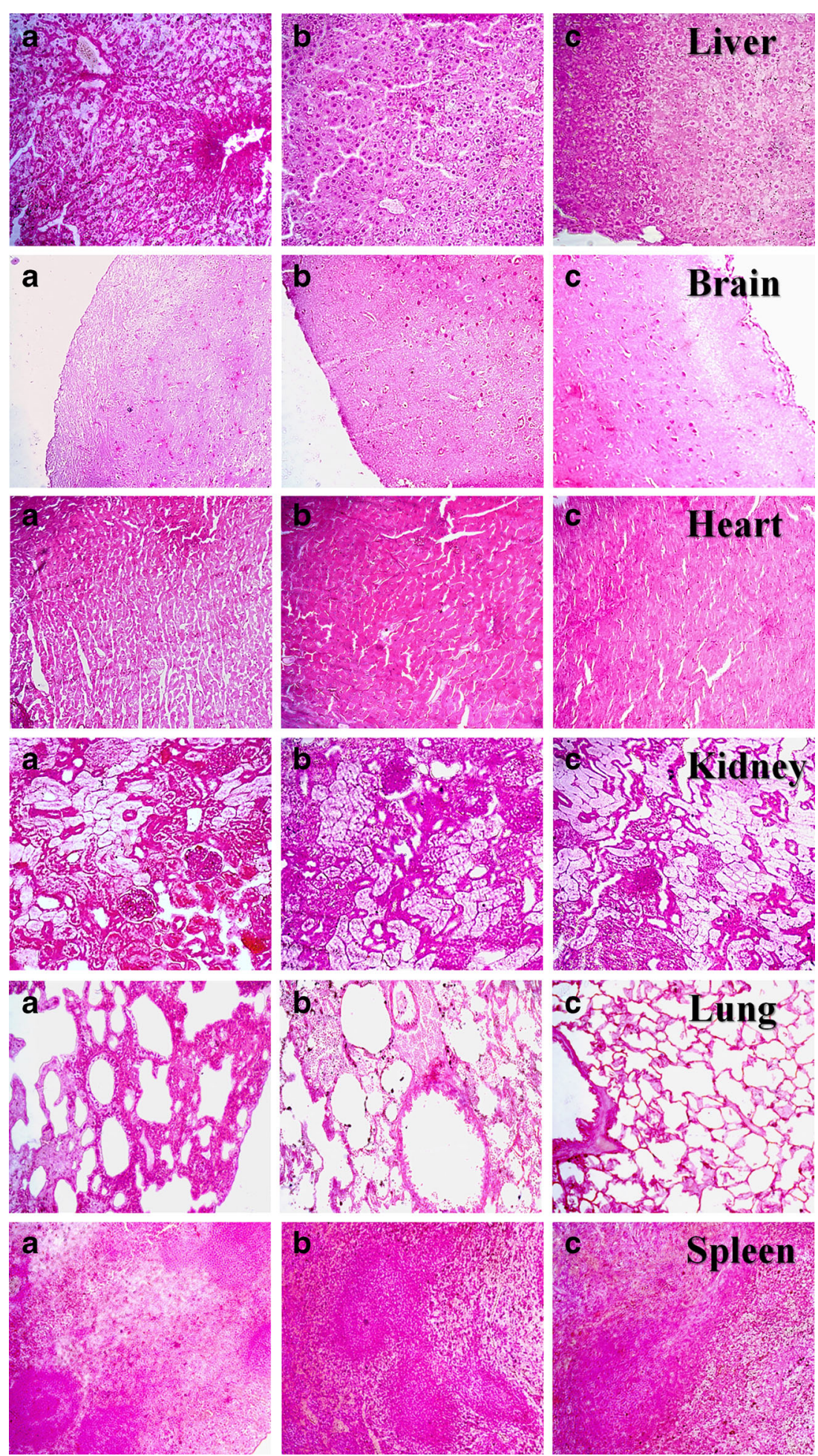

This could be of great benefit to cancer patients by potentially enabling a reduction in the dose administered and eliminating the need for frequent injections of the drug. However caution needs to be taken into account when extrapolating to the human situation from in vitro and rat results. 


\section{REFERENCES}

1. Ding J, Xiao G, Li Y, Cheng Y, Wang N, He C, et al. Efficacious hepatoma-targeted nanomedicine self-assembled from galactopeptide and doxorubicin driven by two-stage physical interactions. Second Symp Innov Polym Control Deliv SIPCD 2012. 2013;169:193-203.

2. Wang J, Xu W, Guo H, Ding J, Chen J, Guan J, et al. Selective intracellular drug delivery from $\mathrm{pH}$-responsive polyion complex micelle for enhanced malignancy suppression in vivo. Colloids Surf B: Biointerfaces. 2015;135:283-90.

3. Chen J, Ding J, Xiao C, Zhuang X, Chen X. Emerging antitumor applications of extracellularly reengineered polymeric nanocarriers. Biomater Sci. 2015;3:988-1001.

4. Shalaby KS, Soliman ME, Casettari L, Bonacucina G, Cespi M, Palmieri GF, et al. Determination of factors controlling the particle size and entrapment efficiency of noscapine in PEG/PLA nanoparticles using artificial neural networks. Int J Nanomedicine. 2014;9: 4953-64.

5. Danquah MK, Zhang XA, Mahato RI. Extravasation of polymeric nanomedicines across tumor vasculature. Adv Drug Deliv Rev. 2011;63:623-39.

6. Steichen SD, Caldorera-Moore M, Peppas NA. A review of current nanoparticle and targeting moieties for the delivery of cancer therapeutics. Eur J Pharm Sci. 2013;48:416-27.

7. Uzgiris EE. Tumor microvasculature: endothelial leakiness and endothelial pore size distribution in a breast cancer model. Breast Cancer Basic Clin Res. 2008; 1:83-90.

8. Lavasanifar A, Samuel J, Kwon GS. Poly(ethylene oxide)-blockpoly(L-amino acid) micelles for drug delivery. Adv Drug Deliv Rev. 2002;54:169-90.

9. Kwon GS, Kataoka K. Block copolymer micelles as longcirculating drug vehicles. Adv Drug Deliv Rev. 1995;16:295-309.

10. Kato K, Chin K, Yoshikawa T, Yamaguchi K, Tsuji Y, Esaki T, et al. Phase II study of NK105, a paclitaxel-incorporating micellar nanoparticle, for previously treated advanced or recurrent gastric cancer. Investig New Drugs. 2012;30:1621-7.

11. Cabral H, Kataoka K. Progress of drug-loaded polymeric micelles into clinical studies. J Control Release. 2014;190:465-76.

12. Takahashi A, Yamamoto Y, Yasunaga M, Koga Y, Kuroda J-I, Takigahira M, et al. NC-6300, an epirubicin-incorporating micelle, extends the antitumor effect and reduces the cardiotoxicity of epirubicin. Cancer Sci. 2013;104:920-5.

13. Soppimath KS, Aminabhavi TM, Kulkarni AR, Rudzinski WE. Biodegradable polymeric nanoparticles as drug delivery devices. J Control Release. 2001;70:1-20.

14. Nakanishi T, Fukushima S, Okamoto K, Suzuki M, Matsumura Y, Yokoyama M, et al. Development of the polymer micelle carrier system for doxorubicin. Proc Int Symp Tumor Target Deliv Syst. 2001;74:295-302.

15. Hobbs SK, Monsky WL, Yuan F, Roberts WG, Griffith L, Torchilin VP, et al. Regulation of transport pathways in tumor vessels: role of tumor type and microenvironment. Proc Natl Acad Sci U S A. 1998;95:4607-12.

16. Prokop A, Davidson JM. Nanovehicular intracellular delivery systems. J Pharm Sci. 2008;97:3518-90.

17. Pitt CG, Gratzl MM, Jeffcoat AR, Zweidinger R, Schindler A. Sustained drug delivery systems. II: factors affecting release rates from poly( $\varepsilon$-caprolactone) and related biodegradable polyesters. J Pharm Sci. 1979;68:1534-8.

18. Shiaw-Guang Hu D, Liu H-J. Effect of soft segment on degradation kinetics in polyethylene glycol/poly(l-lactide) block copolymers. Polym Bull. 1993;30:669-76.

19. Chandra R, Madan J, Singh P, Chandra A, Kumar P, Tomar V, et al. Implications of nanoscale based drug delivery systems in delivery and targeting tubulin binding agent, noscapine in cancer cells. Curr Drug Metab. 2012;13:1476-83.

20. Younes H, Cohn D. Phase separation in poly(ethylene glycol)/ poly(lactic acid) blends. Eur Polym J. 1988;24:765-73.

21. Wu Y, Jiao F, Han S, Fan T, Liu Y, Li W, et al. Construction of amphiphilic copolymer nanoparticles based on hyperbranched Poly (Amine-Ester) and 1,2-Dipalmitoyl-Sn-Glycero-3Phosphoethanolamine as drug carriers for cancer therapy. Nanomed Nanotechnol Biol Med. 201 1;7:945-54.

22. Layre A, Couvreur P, Chacun H, Richard J, Passirani C, Requier $\mathrm{D}$, et al. Novel composite core-shell nanoparticles as busulfan carriers. J Control Release. 2006;111:271-80.

23. Li D, Ding JX, Tang ZH, Sun H, Zhuang XL, Xu JZ, et al. In vitro evaluation of anticancer nanomedicines based on doxorubicin and amphiphilic Y-shaped copolymers. Int J Nanomedicine. 2012;7: 2687-97.

24. Li D, Sun H, Ding J, Tang Z, Zhang Y, Xu W, et al. Polymeric topology and composition constrained polyether-polyester micelles for directional antitumor drug delivery. Acta Biomater. 2013;9: 8875-84.

25. Li T, Han R, Wang M, Liu C, Jing X, Huang Y. Fusiform micelles from nonlinear poly(ethylene glycol)/polylactide copolymers as biodegradable drug carriers. Macromol Biosci. 2011;11:1570-8.

26. Cespi M, Casettari L, Bonacucina G, Giorgioni G, Perinelli DR, Palmieri GF. Evaluation of methoxy polyethylene glycolpolylactide diblock copolymers as additive in hypromellose film coating. Polym Adv Technol. 2013;24:1018-24.

27. Maglio G, Nicodemi F, Conte C, Palumbo R, Tirino P, Panza E, et al. Nanocapsules based on linear and Y-shaped 3-miktoarm starblock peo-pcl copolymers as sustained delivery system for hydrophilic molecules. Biomacromolecules. 2011;12:4221-9.

28. Dahlström B, Mellstrand T, Löfdahl G-G, Johansson M. Pharmakokinetic properties of noscapine. Eur J Clin Pharmacol. 1982;22:535-9.

29. Nayak KP, Brochmann-Hanssen E, Way EL. Biological disposition of noscapine I. Kinetics of metabolism, urinary excretion, and organ distribution. J Pharm Sci. 1965;54:191-4.

30. Perinelli DR, Bonacucina G, Cespi M, Naylor A, Whitaker M, Palmieri GF, et al. Evaluation of P(L)LA-PEG-P(L)LA as processing aid for biodegradable particles from gas saturated solutions (PGSS) process. Int J Pharm. 2014;468:250-7.

31. Li W, Zhang W, Yang X, Xie Z, Jing X. Biodegradable polymersomes from four-arm PEG-b-PDLLA for encapsulating hemoglobin. J Appl Polym Sci. 2014;131.

32. D'Addio SM, Saad W, Ansell SM, Squiers JJ, Adamson DH, Herrera-Alonso M, et al. Effects of block copolymer properties on nanocarrier protection from in vivo clearance. J Control Release. 2012;162:208-17.

33. Kanaoka S, Sueokaw M, Sawamoto M, Higashimur T. Starshaped polymer by living cationic polymerization. VII amphiphilic graft polymers of vinyl ethers with hydroxyl groups: synthesis and host-guest interaction. J Polym Sci Polym Chem Ed. 1993;31: 2513-21.

34. Madan J, Dhiman N, Parmar VK, Sardana S, Bharatam PV, Aneja $\mathrm{R}$, et al. Inclusion complexes of noscapine in $\beta$-cyclodextrin offer better solubility and improved pharmacokinetics. Cancer Chemother Pharmacol. 2010;65:537-48.

35. Ouyang C, Liu Q Zhao S, Ma G, Zhang Z, Song C. Synthesis and characterization of star-shaped poly (lactide-co-glycolide) and its drug-loaded microspheres. Polym Bull. 2012;68:27-36.

36. Yoon K, Kang HC, Li L, Cho H, Park M-K, Lee E, et al. Amphiphilic poly(ethylene glycol)-poly(?-caprolactone) AB2 miktoarm copolymers for self-assembled nanocarrier systems: synthesis, characterization, and effects of morphology on antitumor activity. Polym Chem. 2015;6:531-42. 
37. Fessi H, Puisieux F, Devissaguet JP, Ammoury N, Benita S. Nanocapsule formation by interfacial polymer deposition following solvent displacement. Int J Pharm. 1989;55:R1-4.

38. Leo E, Brina B, Forni F, Vandelli MA. In vitro evaluation of PLA nanoparticles containing a lipophilic drug in water-soluble or insoluble form. Int J Pharm. 2004;278:133-41.

39. Tang J, Liu Z, Zhang Y, Wang L, Li D, Ding J, et al. Fluorofenidone-loaded PLGA microspheres for targeted treatment of paraquat-induced acute lung injury in rats. RSC Adv. 2015;5: 30153-9.

40. Zhang J, Men K, Gu Y, Wang X, Gou M, Guo G, et al. Preparation of core cross-linked PCL-PEG-PCL micelles for doxorubicin delivery in vitro. J Nanosci Nanotechnol. 201 1;11:5054-61.

41. Endres TK, Beck-Broichsitter M, Samsonova O, Renette T, Kissel TH. Self-assembled biodegradable amphiphilic PEG-PCL-PEI triblock copolymers at the borderline between micelles and nanoparticles designed for drug and gene delivery. Biomaterials. 2011;32: 7721-31.

42. Madan J, Dhiman N, Sardana S, Aneja R, Chandra R, Katyal A. Long-circulating poly(ethylene glycol)-grafted gelatin nanoparticles customized for intracellular delivery of noscapine: preparation, invitro characterization, structure elucidation, pharmacokinetics, and cytotoxicity analyses. Anti-Cancer Drugs. 201 1;22:543-55.

43. Sebak S, Mirzaei M, Malhotra M, Kulamarva A, Prakash S. Human serum albumin nanoparticles as an efficient noscapine drug delivery system for potential use in breast cancer: preparation and in vitro analysis. Int J Nanomedicine. 2010;5:525-32.

44. Chollet DF, Ruols C, Arnera V. Determination of noscapine in human plasma using solid-phase extraction and high-performance liquid chromatography. J Chromatogr B Biomed Appl. 1997;701: 81-5.

45. Abulateefeh SR, Saeed AO, Aylott JW, Chan WC, Garnett MC, Saunders BR, et al. Facile synthesis of responsive nanoparticles with reversible, tunable and rapid thermal transitions from biocompatible constituents. Chem. Commun. 2009;6068-70.

46. Kumar S, Kumar R, Dwivedi A, Pandey AK. In vitro antioxidant, antibacterial, and cytotoxic activity and in vivo effect of Syngonium podophyllum and Eichhornia crassipes leaf extracts on isoniazid induced oxidative stress and hepatic markers. BioMed Res Int. 2014;2014:459452.

47. Skehan P, Storeng R, Scudiero D, Monks A, McMahon J, Vistica $\mathrm{D}$, et al. New colorimetric cytotoxicity assay for anticancer-drug screening. J Natl Cancer Inst. 1990;82:1 107-12.

48. Winter CA, Flataker L. Toxicity studies on noscapine. Toxicol Appl Pharmacol. 1961;3:96-106.

49. Aneja R, Dhiman N, Idnani J, Awasthi A, Arora SK, Chandra R, et al. Preclinical pharmacokinetics and bioavailability of noscapine, a tubulin-binding anticancer agent. Cancer Chemother Pharmacol. 2007;60:831-9.

50. Zhang Y, Huo M, Zhou J, Xie S. PKSolver: an add-in program for pharmacokinetic and pharmacodynamic data analysis in microsoft excel. Comput Methods Prog Biomed. 2010;99:306-14.

51. Moura JA, Valduga CJ, Tavares ER, Kretzer IF, Maria DA, Maranhão RC. Novel formulation of a methotrexate derivative with a lipid nanoemulsion. Int J Nanomedicine. 201 1;6:2285-95.

52. Sachse C, Henkel E. An evaluation of the CELL-DYN 17000® haematology analyser: automated cell counting and three-part leucocyte differentiation. Clin Lab Haematol. 1996;18:171-80.

53. Allen C, Maysinger D, Eisenberg A. Nano-engineering block copolymer aggregates for drug delivery. Colloids Surf B: Biointerfaces. 1999;16:3-27.
54. Gong J, Chen M, Zheng Y, Wang S, Wang Y. Polymeric micelles drug delivery system in oncology. J Control Release. 2012;159: 312-23.

55. Nishiyama N, Kataoka K. Current state, achievements, and future prospects of polymeric micelles as nanocarriers for drug and gene delivery. Pharmacol Ther. 2006;112:630-48.

56. Stolnik S, Illum L, Davis SS. Long circulating microparticulate drug carriers. Adv Drug Deliv Rev. 1995;16:195-214.

57. Zamani S, Khoee S. Preparation of core-shell chitosan/PGL-PEG triblock copolymer nanoparticles with $\mathrm{ABA}$ and $\mathrm{BAB}$ morphologies: effect of intraparticle interactions on physicochemical properties. Polymer. 2012;53:5723-36.

58. Roos WH, Bruinsma R, Wuite GJL. Physical virology. Nat Phys. 2010;6:733-43.

59. Zhang L, Hu Y, Jiang X, Yang C, Lu W, Yang YH. Camptothecin derivative-loaded poly(caprolactone-co-lactide)-b-PEG-bpoly(caprolactone-co-lactide) nanoparticles and their biodistribution in mice. J Control Release. 2004;96:135-48.

60. Manisha K, Maheshwar S, Madhuri S. Encapsulation of berberine in nano-sized PLGA synthesized by emulsification method. ISRN Nanotechnol. 2012;2012:1-9.

61. Madan J, Gundala SR, Kasetti Y, Bharatam PV, Aneja R, Katyal A, et al. Enhanced noscapine delivery using estrogen-receptortargeted nanoparticles for breast cancer therapy. Anti-Cancer Drugs. 2014;25:704-16.

62. Gerweck LE, Seetharaman K. Cellular $\mathrm{pH}$ gradient in tumor versus normal tissue: potential exploitation for the treatment of cancer. Cancer Res. 1996;56:1194-8.

63. de Graaf AJ, Boere KWM, KemminkJ, Fokkink RG, van Nostrum CF, Rijkers DTS, et al. Looped structure of flowerlike micelles revealed by $1 \mathrm{H}$ NMR relaxometry and light scattering. Langmuir. 2011;27:9843-8.

64. Xiao RZ, Zeng ZW, Zhou GL, WangJJ, Li FZ, Wang AM. Recent advances in PEG-PLA block copolymer nanoparticles. Int J Nanomedicine. 2010;5:1057-65.

65. Owens III DE, Peppas NA. Opsonization, biodistribution, and pharmacokinetics of polymeric nanoparticles. Int J Pharm. 2006;307:93-102.

66. Gibaldi M, Weiner ND. Biphasic elimination of noscapine. J Pharm Sci. 1966;55:769-71.

67. Madan J, Pandey RS, Jain UK, Katare OP, Aneja R, Katyal A. Sterically stabilized gelatin microassemblies of noscapine enhance cytotoxicity, apoptosis and drug delivery in lung cancer cells. Colloids Surf B: Biointerfaces. 2013;107:235-44.

68. Madan J, Pandey RS, Jain V, Katare OP, Chandra R, Katyal A. Poly (ethylene)-glycol conjugated solid lipid nanoparticles of noscapine improve biological half-life, brain delivery and efficacy in glioblastoma cells. Nanomed Nanotechnol Biol Med. 2013;9: 492-503.

69. Yadav AK, Mishra P, Jain S, Mishra P, Mishra AK, Agrawal GP. Preparation and characterization of HA-PEG-PCL intelligent core corona nanoparticles for delivery of doxorubicin. J Drug Target. 2008; 16:464-78.

70. Shuai X, Ai H, Nasongkla N, Kim S, GaoJ. Micellar carriers based on block copolymers of poly( $\varepsilon$-caprolactone) and poly(ethylene glycol) for doxorubicin delivery. J Control Release. 2004;98:415-26.

71. Xin H, Sha X, Jiang X, Zhang W, Chen L, Fang X. Antiglioblastoma efficacy and safety of paclitaxel-loading Angiopepconjugated dual targeting PEG-PCL nanoparticles. Biomaterials. 2012;33:8167-76. 